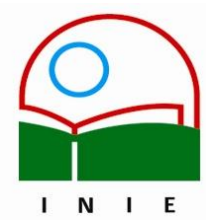

Actualidades Investigativas en Educación

Revista Electrónica publicada por el

Instituto de Investigación en Educación

Universidad de Costa Rica

ISSN 1409-4703

http://revista.inie.ucr.ac.cr

COSTA RICA

\title{
LA RECEPCIÓN DE LA OBRA DE LIEV VIGOTSKY EN LAS DÉCADAS DE 1980 Y 1990 EN ARGENTINA \\ THE RECEPTION OF LIEV VIGOTSKY'S ARGENTINA
}

\author{
Volumen 9, Número Especial
}

pp. 1-24

Este número se publicó el 15 de noviembre 2009

Andrés Gomel

Marta Alicia Tenutto Soldevilla

La revista está indexada en los directorios:

LATINDEX, REDALYC, IRESIE, CLASE, DIALNET, DOAJ, E-REVIST@S,

La revista está incluida en los sitios:

REDIE, RINACE, OEI, MAESTROTECA, PREAL, HUASCARAN, CLASCO

Los contenidos de este artículo están bajo una licencia Creative Commons 


\title{
LA RECEPCIÓN DE LA OBRA DE LIEV VIGOTSKY EN LAS DÉCADAS DE 1980 Y 1990 EN ARGENTINA
} THE RECEPTION OF LIEV VIGOTSKY'S ARGENTINA

\author{
Andrés Gomel ${ }^{1}$ \\ Marta Alicia Tenutto Soldevilla²
}

\begin{abstract}
Resumen: En este texto, procuraremos abordar las huellas que ha dejado la recepción de la obra de Liev Vigotsky en el campo pedagógico argentino en las décadas de 1980 y 1990. Se pondrán a disposición algunas categorías, así como los modos en que éstas fueron abordadas. La decisión de trabajar tanto la obra de Liev Vigotsky como la de Jean Piaget desde la teoría de la recepción, está basada en que el primero fue abordado en la década de 1990 en relación con el segundo y ambos fueron ubicados dentro del amplio espectro de posiciones subsumidas bajo la denominación "constructivismo" en las décadas citadas.
\end{abstract}

Palabras claves: VIGOTSKY, TEORIA DE LA RECEPCIÓN, CONSTRUCTIVISMO, TEORIA SOCIOHISTÓRICA

Abstract: In this text, we will attempt to discuss the marks left by the reception of Liev Vigotsky's work into the pedagogical field in general and particularly of Argentina during the '80s and '90s. A selection of categories will be presented, as well as the ways in which these were approached. The decision to elaborate on Liev Vigotsky's work -as well as on Jean Piaget's- from the approach of its introduction into the Argentinian pedagogical field is based on the fact that the former was discussed in the '80s and '90 in relation to the latter and both placed within the wide spectrum of views labeled during that decade as "constructivism".

Key words: VIGOTSKY, RECEPTION THEORY, CONSTRUCTIVISM, SOCIOHISTORICAL THEORY

\footnotetext{
${ }^{1}$ Magister y Especialista en Educación, de la Universidad de San Andrés, Argentina. Licenciado en Psicología y Profesor de Psicología, de la Universidad de Buenos Aires. Diploma en Constructivismo y Educación (FLACSO). Profesor e investigador de la Universidad de Buenos Aires, Argentina. Docente UADE, UAI. Dirección electrónica: andresgomel@hotmail.com

${ }^{2}$ Magister y Especialista en Educación de la Universidad de San Andrés, Argentina., Especialista en Psicología educacional (Col. de psicólogos de la prov. de Bs. As.). Licenciada en Psicología de la Universidad de Buenos Aires. Licenciada y Profesora en Ciencias de la educación, de la Universidad de Morón (UM). Profesora para la enseñanza Primaria. Autora de "Herramientas de evaluación en el aula". Docente de la UP y de la Universidad Favaloro. Investigadora. Coordinadora de los Profesorados de Cs. Sociales (UBA). Dirección electrónica: mtenutto@gmail.com
}

Artículo recibido: 3 de junio, 2009

Aprobado: 19 de octubre, 2009 


\section{Introducción}

Es preciso recorrer el nuevo territorio con nuevas preguntas y nuevos ojos (...) construir nuevos instrumentos de conocimiento para levantar acta de la nueva tierra, describir sus configuraciones, reunir vestigios de pensamiento casi perdidos 0 en todo caso olvidados, en fin, excavar bajo arbustos y cenizas incluso bajo lujosos palacios ... (Alvarez-Uría, 1998, p. 13)

En la década de 1980 Latinoamérica asiste al regreso de los gobiernos democráticos. Este hecho abre la puerta a la inclusión en el campo educativo de diversos autores que se encontraban, hasta ese momento, desterrados. Es en ese marco cuando la obra de Liev $^{3}$ Vigotsky logra ingresar con fuerza al campo de la educación. $Y$ en la década siguiente, instalados ya los gobiernos democráticos, se concretarán diversas reformas educativas que instalarán a Liev Vigotsky ubicado en la línea del "constructivismo". El "Constructivismo" se rerigió como un significante destacado en esas reformas en tanto parece emerger como organizador de prácticas y representaciones de los y las docentes. En este texto, procuraremos abordar las huellas que su recepción ha dejado en el campo pedagógico argentino en las décadas mencionadas. Se pondrán a disposición algunas categorías así como los modos en que éstas fueron abordadas.

\section{Desarrollo del tema}

\subsection{Proposición}

\subsubsection{Tras los pasos de los autores}

En este texto analizaremos, desde la teoría de la recepción, las obras de Liev Vigotsky y Jean Piaget en el campo pedagógico argentino, en tanto el primero fue abordado en la década de 1990 en relación con el segundo, y ambos fueron ubicados dentro del amplio espectro de posiciones subsumidas bajo la denominación "constructivismo" en esa década.

3 Según la indagación realizada por Guillermo Blanck el autor se llama Liev que significa León (al igual que Tolstoi). According to Guillermo Blanck's research, the author's name is Liev, which jeans Lion (the same as Tolstoi) 
Optamos por la teoría de la recepción, porque esta propuesta remite a las lecturas de una determinada obra que se realizan en un tiempo y lugar, así como a las formas que adoptan tales lecturas, a los grados de aceptación de la obra y a las ideas que producen los "lectores" ${ }^{4}$ sobre la misma. Por lo tanto, ese tipo de análisis permite capturar la significación que se le otorga a la obra en cuestión. Todo estudio de la recepción de una obra implica por tanto una constante -el texto- y distintas variables: las distintas lecturas que suscita en el público, más o menos heterogéneo. En este sentido, Silvia Parrat-Dayan (1998) propone tres objetivos para definir este trabajo: comprender la significación de la obra de un "autor", situar el contexto que permite su recepción e identificar, cuando sea posible, la respuesta del autor a las lecturas.

Cuando la lectura de una determinada obra se hace en el momento de su producción, se trata de una relación de tipo sincrónica entre un autor y un "lector". Esta lectura, sin embargo, está apoyada sobre elementos diacrónicos, esto es, todas las lecturas anteriores que realizó quien lee, y que definen su lectura. Este elemento toma en cuenta, además, la importancia del contexto sociocultural en el cual se sitúan la obra y las personas que la leen.

La perspectiva de la lectura diacrónica, por su parte, permite capturar tanto el contexto específico de una época, como las posibles reinterpretaciones que se deben a la transformación de las ideas. Esto ayuda a comprender, por ejemplo, que las críticas que recibe una obra a lo largo del tiempo están ligadas a la concepción de lector propia de cada época histórica, más que a una aparente objetividad de la obra misma. Es en esta interacción que se produce un diálogo entre autores y personas que leen. ${ }^{5}$ En este sentido, a los fines de entender las recepciones de las diferentes obras, interesa conocer aquellas que las precedieron, ya que las obras nunca se presentan como una novedad absoluta sino que

4 Nos interesa remarcar que en este trabajo se utiliza lenguaje no sexista. Las referencias a personas, colectivos o cargos citados en los textos en género masculino refieren a categorías teóricas, por lo que no han sido modificadas. En el caso indicado, recuperamos las categorías de "lector" y "autor" tal como las trabaja la Teoría de la Recepción.

5 Santagada (1999) sostiene que la cultura occidental ha adjudicado, desde tiempos remotos, enorme valor a la interpretación y comprensión de textos escritos. Por esa razón, las normas para la exégesis correcta de textos se impusieron a lo largo del tiempo desde instituciones como la escuela, la crítica especializada o los diferentes círculos intelectuales, que pretendieron dictaminar acerca del sentido exacto de los textos modelando así las prácticas culturales. Según este autor, es indudable la función de las agencias que prescriben lecturas preferenciales sobre los textos, no que tales agencias logren mantener sus imposiciones de un modo incontestable y permanente. 
evocan siempre otras lecturas realizadas. De esta forma, el "lector" tiene una predisposición diferente frente a cada lectura.

En otro orden de cosas, resulta relevante la discusión acerca del concepto de "horizonte de expectativa”. Parrat-Dayan, citando a Hans Robert Jauss (1982), lo define como un sistema de referencia representado por el conjunto de conductas, conocimientos, ideas preconcebidas que encuentra una obra en el momento de su aparición y a través de la cual es valorada. Según Jauss, por lo tanto, la figura del lector estaría inscripta en la obra misma, en tanto el contexto de producción de la obra deja sus huellas sobre el texto, además de que presupone un interlocutor con el que dialogar. Por su parte, Parrat-Dayan sostiene que en el imaginario social quienes leen asocian sus lecturas está ligado al concepto de horizonte de expectativas, esto es, lo que la cultura ofrece en un momento determinado. Por lo tanto, lo fundamental para la autora es sostener una mirada dialógica frente al fenómeno, capaz de articular las expectativas definidas socialmente con los aspectos sociales concretos.

Este concepto es discutido por Caruso y Fairstein (1997), quienes consideran que el concepto de "horizonte de expectativas" es "ahistoricista", pues entienden que determina inmanentemente ${ }^{6}$ al lector. Para estos autores, es fundamental rescatar la figura de un lector articulador de la obra, lo cual refiere a una profunda idea de historicidad en relación con la misma. Por eso, proponen reemplazar esta idea por los conceptos de "fantasía social" y "dimensión imaginaria" del lector, para definir -desde una perspectiva histórica- los usos y lecturas de cada obra, de manera tal de recuperar en el análisis de las lecturas las luchas políticas, los imaginarios educacionales, los espacios sociales que ocupa o cree ocupar quien lee, entre otros elementos. En este sentido, no interesa tanto la fidelidad de la lectura respecto de la obra original, sino que es el mismo acto de lectura el que brinda una caracterización del lector.

6 Maldonado Veloza retoma el planteo de Karl Popper quien distinque entre crítica inmanente y crítica trascendente. Una teoría se ataca inmanentemente desde dentro y según sus propios supuestos y presuposiciones y cree que quienes han sostenido que la crítica inmanente no es útil porque "como no puede hacer más que señalar las inconsistencias lógicas dentro de la teoría criticada, nunca tiene éxito contra una teoría consistente" (Popper, 1985, p. 69). Pero ha respondido que tales exponentes están equivocados. 
Surge entonces la idea del lector como articulador, como "sede" de las lecturas. Los lectores se presentan como articuladores de las lecturas dado que conectan las mismas con sus tradiciones, visiones políticas y predisposiciones culturales, estableciendo la lectura como una práctica social articulatoria (Laclau y Mouffe, 1987, citado en Caruso y Fairstein, 1997). La recepción se considera entonces como el proceso que define un espacio donde los sujetos construyen su historia, identidades y estrategias.

Desde esta propuesta se ha estudiado en mayor medida la inclusión del llamado "piagetismo" en la Argentina. Se puede mencionar, en primer lugar, la investigación de Marcelo Caruso y Gabriela Fairstein (1997), quienes se proponen estudiar a Jean Piaget y la escuela ginebrina en tanto obra, y no en tanto textos. Estudian qué es lo que quienes integran el campo pedagógico realizan con el corpus teórico piagetiano y cómo y de qué forma lo articulan a otros enunciados y propuestas pedagógicas. Se busca, en este gesto, desmitificar al "lector", aunque respetando el corpus de referencia. Por lo tanto, no les interesa la fidelidad en la lectura de la obra, sino la posibilidad de comprender el acto de lectura mismo como posibilidad de caracterización del lector. Existe acuerdo en pensar que la recepción de una determinada obra y su reconstrucción por parte del lector debe ser analizada teniendo en cuenta su experiencia anterior: lo que un lector espera de un texto es distinto si se trata de una primera aproximación o si se trata de una lectura "informada". En relación con esto, por último, es importante comprender la recepción de una obra situándola en un contexto científico más amplio. Este contexto crea en el lector una imagen anticipatoria acerca del contenido textual, la cual está directamente relacionada con sus lecturas anteriores. Esta imagen puede resultar más o menos sólida, en relación con las tradiciones intelectuales que anteceden las apariciones de las obras y que van moldeando la posibilidad de recepción por parte de quienes leen.

Pero no solo Marcelo Caruso y Gabriela Fairstein (1997) estudiaron la recepción de la teoría piagetiana en el campo pedagógico argentino sino que, también, hay que mencionar como referente que indagó las vinculaciones de las teorías psicológicas y campo educativo a partir de la categoría de recepción a Silvia Parrat-Dayan (1998) Esta autora sostiene que

el estudio de la recepción de las obras de un autor puede tener objetivos diferentes. Uno de esos objetivos es comprender mejor las ideas del autor en cuestión. Otro objetivo consiste en tratar de saber qué es lo que provoca la aceptación o el rechazo 
de la obra en una época dada de la historia o a lo largo de toda la vida del autor. Por otro lado, la idea que un lector produce a partir de un texto puede o no estar de acuerdo con la concepción del autor, que puede o no responder. (1997, p. 145)

\subsubsection{Sobre las derivaciones educativas}

En primer lugar, y para comprender los usos y derivaciones que se han realizado de la teoría sociohistórica en el campo educativo, puede ser de utilidad revisar en primer lugar los derroteros que han seguido los usos de la teoría de Jean Piaget. Marcelo Caruso y Gabriela Fairstein reconocen que a partir de la década de 1930 se puede notar la presencia de lo que hoy se da en llamar "constructivismo" en el campo pedagógico argentino. Fue entonces cuando se registran las primeras referencias a Jean Piaget en la provincia de Buenos Aires, empleándose la obra de este autor como "oportunidad para la normalización" (Caruso y Fairstein, 1997, p. 168) e iniciando así una lectura que se halla atravesada por el espiritualismo en boga. Es el momento en que se mezclaron postulados de la escuela nueva y del normalismo en el marco de reformas de tinte autoritario. Esto fue característico tanto de la reforma educativa bonaerense de 1937, como de los manuales de la década de 1940.

En la década siguiente, Piaget fue invocado en los manuales de pedagogía, como el de Luzuriaga, con el fin de conocer las disposiciones de la niñez (Caruso y Fairstein, 1997). Pero fue en la década de 1960 cuando Piaget ingresó a las aulas de la universidad, a través de materias como "Didáctica del jardín de infantes y de primaria", en la carrera de Ciencias de la educación, o de "Psicología General II y III" en Psicología. Paralelamente, se lo difundió en las escuelas normales, se conformaron grupos de estudio, se fundaron instituciones de nivel inicial inspiradas en sus postulados y se publicaron textos referidos a este nivel que lo adoptaron como marco de referencia. La clave de lectura de esta obra es de "fascinación estructuralista", la cual "parecía compatible y operativa con una pedagogía de corte tecnicista que veía en la planificación de la enseñanza según objetivos una racionalización de las prácticas intuitivas" (Caruso y Fairstein, 1997, p. 181).

Entre 1971 y 1976 se elaboraron contenidos en matemática en el marco del piagetianismo, produciéndose el habitualmente criticado "aplicacionismo" de una propuesta psicológica, sin intermediación al ámbito de la pedagogía (Coll, 1991). Fue un tiempo en el cual las investigaciones de base piagetiana poseían también fuertes vínculos con la política Volumen 9, Número Especial, Año 2009, ISSN 1409-4703 
radicalizada, por lo que fue en este contexto cuando se difundieron sus hipótesis epistemológicas.

Entre los años 1976 y 1983 se asistió en Argentina a una política de prohibición (y de muerte) donde Piaget quedó ubicado en línea con otros "fantasmas" que debían ser desterrados de la Universidad (Marx, Freud) y se dispersaron quienes habían abierto la puerta a la lectura epistemológica y a la psicogénesis de los contenidos escolares, quedando en la transmisión tecnicista el corpus piagetiano asimilado a una didáctica altamente prescriptiva.

A partir de 1980 se produjo un debate en torno a los aportes respectivos de Piaget y Vigotsky, el cual condujo a los investigadores e investigadoras a trabajar sobre las preguntas que guían las investigaciones de Piaget y de Vigotsky, a fin de desandar el camino en el cual se los presentaba como autores necesariamente complementarios. ${ }^{7}$ En las huellas de estas experiencias se produjo la incorporación del constructivismo a la formación docente y particularmente se ubica al constructivismo como significante privilegiado en la reforma producida en Argentina en la década de los 90. Coll sostiene que

la visión constructivista del psiquismo humano impregna por completo la psicología de la educación (...). La idea original del constructivismo es que el conocimiento y el aprendizaje son, en buena medida, el resultado de una dinámica en la que las aportaciones del sujeto al acto de conocer y aprender juegan un papel decisivo. (Coll; 2001, p. 157)

Según este autor a partir de la segunda mitad de la década del 70 comienza a disminuir la influencia de la teoría piagetiana en el campo educativo aunque, es importante aclarar, mantiene su importancia-, por lo que otras teorías, calificadas o autocalificadas habitualmente como constructivistas, comienzan a disputarle protagonismo, especialmente aquellas vinculadas al enfoque del procesamiento de la información, la teoría sociohistórica y los enfoques neovigotskianos. Por su parte, el investigador argentino Ricardo Baquero (1996) coincide en que resulta habitual entender el crecimiento en el interés por la obra

7 Vigotsky pretendía alcanzar una explicación sociohistórica de la constitución de las funciones psíquicas, en cambio, la pregunta central para Piaget gira alrededor del pasaje de un estado de menor conocimiento a uno de mayor conocimiento (Castorina, 1996, p.15). 
vigotskiana a partir del declinar de las obras de otros autores, especialmente Piaget, pero advierte que esto no implica necesariamente la superación de una perspectiva por otra, dado que tanto las preguntas que se hace cada teoría como los problemas que recorta no son idénticos (también Castorina, 1996).

El paradigma vigotskiano puede pensarse como relativamente nuevo en occidente, dado que su lectura sistemática comenzó recién a fines de la década de los sesenta (aunque, como sabemos, la obra se ubica principalmente desde fines de los años 20 y comienzos de los años 30).

Los planteos y derivaciones de la obra vigotskiana en educación deberían ser analizadas tomando en cuenta que Vigotsky, a diferencia de Piaget, trabajó en profundidad sobre cuestiones referidas al ámbito educativo. Esto produjo un marcado interés de parte de los educadores y educadoras por su producción, en gran medida debido a la posibilidad de encontrar en ella heurísticos para analizar los procesos educativos.

\subsection{Argumentación}

\subsubsection{Sobre los "usos" de la teoría sociohistórica}

Las producciones de los investigadores e investigadoras no son obras monolíticas y no se mantienen inalterables a la largo de la vida de los mismos. Jean Piaget, Liev Vigotsky, Jerome Bruner o David Ausubel tomaron lo que su época les proporcionó y en numerosas ocasiones modificaron sus perspectivas de acuerdo a los marcos teóricos que fueron incorporando. A menudo cambiaron las respuestas que alguna vez dieron a aquello que se habían planteado inicialmente y en otras oportunidades llegaron a modificar sus preguntas a lo largo de su obra (en tanto fueron cambiando su modo de ver el mundo y al hacerlo introdujeron cambios en sus teorías). Se trata de mostrar una perspectiva de los autores trabajados que dé cuenta del proceso de gestación de las ideas, en lugar de considerar su obra como un producto que fue anticipado desde sus inicios, para mostrar la complejidad de los discursos a los que se han enfrentado los y las docentes en los últimos años.

Los usos de la teoría de Vigotsky en educación no han sido en modo alguno homogéneos. En la mayoría de los casos, los trabajos de raigambre vigotskiana se definen como tales Volumen 9, Número Especial, Año 2009, ISSN 1409-4703 
fundamentalmente a partir de la apelación a varias de las hipótesis centrales de la teoría, más que en virtud de compartir un ideario pedagógico unívoco. Podemos decir que esta teoría ha sido utilizada en modos de alguna manera paradójicos, o que ha enfrentado tensiones en relación al lugar en el que situar el énfasis de los trabajos realizados en el campo pedagógico (Baquero, 1998).

Los primeros trabajos o elaboraciones sobre la teoría de Vigotsky que se realizaron en relación a prácticas de enseñanza tuvieron, según Hatano (1991), un uso o sesgo donde primaron básicamente aspectos instruccionales. El marco vigotskiano, leído desde este foco, resultaba compatible con la enseñanza tradicional. Este sesgo en el uso de la teoría remite a la importancia de analizar los aspectos reproductivistas que pudiera tener una pedagogía centrada en la teoría sociohistórica, esto es, una pedagogía que implique estrategias de enseñanza con un marcado tono directivista. Es lo que Baquero llama "pedagogía centrada en el adulto" o de sesgo adultocéntrico (1998).

De manera sintética, diremos a comienzos de la década de 1980 se inicia una lectura más compleja de esta teoría, que dará lugar a los trabajos de diversos autores y autoras como Cazden (1998) en el área del análisis del discurso en el aula y la apropiación de la lengua escrita, Edwards y Mercer (1988), Newman, Griffin y Cole (1991) vinculados a los análisis de las características de la organización de las actividades y la definición de tareas en las prácticas de enseñanza. Hacia fines de esa década surgen los trabajos sobre aprendizaje guiado y cooperativo y enseñanza recíproca (Rogoff, 1993; Tudge, 1993) y los trabajos sobre la enseñanza de la lectura y la escritura de Goodman y Goodman (1993), y en lengua española trabajos como los de Onrubia (1993), entre otros. También cabe mencionar los trabajos en el área del desarrollo de los conceptos científicos en contextos de enseñanza, como los de Hedegaard (1993).

\subsubsection{La obra y las reformas educativas}

Los trabajos señalados en el apartado anterior se realizan en la misma década en la que en Argentina, en el marco de la reforma del Estado, se lleva adelante la llamada "Reforma 
Educativa" ${ }^{8}$. Se trata de un momento donde se procura que Argentina, así como el resto de Latinoamérica, ${ }^{9}$ encare un proceso de reestructuración, de reducción de personal y de descentralización político-administrativa (Cunill Grau, 1997). En el campo de la educación, la reforma fue incluida en la agenda de gobierno, iniciándose un conjunto de procesos dirigidos a descentralizar y transformar los sistemas educativos. En sintonía con los lineamientos emanados de las políticas educativas latinoamericanas, se instó a que los y las docentes actualicen sus saberes, se "perfeccionen” y se “conviertan” (Braslavsky, Gvirtz, 2001).

La reforma de la década de 1990 fue difundida a través de una política basada en documentos. El hecho de que este proceso se llevara a cabo a través de textos escritos da cuenta de los presupuestos teóricos que sostenían quienes llevaban adelante la reforma, la importancia dada a la cultura letrada y el descrédito otorgado a la cultura empírico-práctica ${ }^{10}$. El cuerpo docente debió enfrentarse a lecturas desprovistas de "texturas", de interrogantes, de algo que pudiera interpelarlos, en cambio se ofreció un saber en cual se les pedía que fueran personas críticas, pero que recibieran esos materiales acríticamente ${ }^{11}$. Si bien no constituye parte de los objetivos de este trabajo el análisis de los materiales entregados a los y las docentes tomaremos, a modo de ejemplo, un módulo realizado en la provincia de Buenos Aires (Argentina) durante las capacitaciones realizadas en esa década.

En este caso, cuando se desarrollan las teorías del aprendizaje, éstas son incluidas en las Explicaciones constructivistas del aprendizaje se ubica el surgimiento de éstas en Europa y se las caracteriza como organicistas y estructuralistas. Además, se explicita que "este enfoque tiene implicancias generales en la concepción de aprendizaje" y por lo define "por

8 En el año 1993 se sanciona la primera ley que pretende legislar sobre la totalidad el sistema educativo: la Ley Federal de educación № 24.195

9 Las leyes se sancionan en los siguientes años: Chile: 1991, Paraguay: 1992, Argentina: 1993, México: 1993, Colombia: 1994, República Dominicana: 1997, Brasil: 2000 y Perú: 2001.

${ }_{10}$ Viñao diferencia: la cultura del "conocimiento experto" o cultura científica de la educación generada en el mundo académico universitario, la cultura "político institucional", asociada al discurso o lenguaje normativo, producida por los entornos administrativos y burocráticos y la cultura "empírico práctica" elaborada por los enseñantes en el ejercicio de su profesión, que constituye la memoria corporativa de los docentes".(Viñao; 2001, p. 42)

11 A modo de ejemplo, una frase que figura en el Módulo de capacitación docente de la provincia de Buenos Aires "Quienes estamos frente al aula, frente a los alumnos sabemos bien que un determinado puntaje nos habilita para estar parados frente a una clase, pero que a la hora final de la verdad, delante de esos ojos oídos y voces ávidas de aprender, el titulo y la dignidad de Maestra tiene una sola llave: el conocimiento" 
oposición al conductismo"12 En los títulos de las dos primeras aportaciones aparecen determinadas teorías (Gestalt y Psicología Genética) en tanto que en las dos segundas figuran los nombres de sus iniciadores (Vigotsky y Ausubel). En el apartado sobre las ideas de Vigotsky se lo presenta como un "investigador que desarrolló sus ideas simultáneamente con Piaget", con quien tuvo una "célebre polémica". Se compara a este autor con el anterior en tanto ambos consideran necesaria la instrucción formal para que la reestructuración se produzca y se favorezca el aprendizaje, además de diferenciar estrategia de enseñanza de estrategia de aprendizaje, se desarrollan conceptos como: aprendizaje significativo y construcción individual y social del conocimiento. En la apreciación crítica se reconoce que esta teoría ha sido formulada recientemente, que valoriza la enseñanza, al cuerpo docente y lo relativo al aprendizaje significativo. Además, no presentan las formas de enseñanza como dicotómicas.

En la investigación realizada por Marta Tenutto acerca de qué significados le atribuían quienes enseñan en los profesorados al constructivismo se arriba a que, en el marco de la presión reformadora de la década de los 90, el cuerpo de profesores procuró apropiarse de los nuevos saberes y se aproximaron a las nuevas propuestas reduciendo lo nuevo a lo conocido, despliegue táctico con lo que respondieron a la estrategia reformadora ${ }^{13}$. Ésta se dirigió a los profesores y profesoras proponiendo la modificación de las prácticas a través de - entre otras cuestiones - la incorporación de nuevas nominaciones y conocimientos, pero aquellos conformaron significaciones propias a las otorgadas por las propuestas orgánicas y estratégicas de reforma. La táctica les permitió producir significaciones discrepantes con respecto a aquéllas propuestas, donde tuvieron un fuerte peso las situaciones personales, institucionales y profesionales.

12 Dirección General de cultura y educación de la provincia de Buenos Aires (1995) Módulo 3, La Plata, p. 40.

${ }_{13}$ La estrategia es la ciencia de los movimientos guerreros fuera del campo de visión de enemigo (Von Bulow) citado por de Certeau, 1996, p. 43, en tanto la táctica constituye el interior del campo, como lugar del poder y de la voluntad propios (de Certeau, 1996, p. 42). 
La dinámica de la reforma y su recepción puede ser analizada bajo la óptica de estrategia y táctica en función de sus posibilidades comunicativas. La reforma ${ }^{14}$ fue propiciada desde una política documentalista y estuvo sostenida en la escritura. Los módulos de capacitación docente de la provincia de Buenos Aires procuraron instalar al constructivismo como parte del discurso pedagógico, ubicándolo en el polo del "deber ser", haciéndolo jugar, de este modo, como opuesto al conductismo, casi como sinónimo de la escuela existente y que se desea cambiar.

\section{Discusión}

Resulta necesario retomar los aportes de Silvia Parrat-Dayan en relación con el concepto de "contexto científico amplio" ya que éste resulta fructífero en tanto marco para la comprensión de los cambios que se van produciendo en los usos de las teorías, conceptos y textos psicológicos. Un análisis de tipo diacrónico, como el que proponemos aquí, permite comprender cuál es el tipo de lectura que se realiza de las teorías en el período histórico contemplado, comparar los resultados con las lecturas hechas en otros períodos e identificar las lecturas previas de cada tradición académica que estarían contribuyendo a la creación de una imagen anticipatoria.

\subsection{Modos de recepción de la teoría vigotskiana}

En el marco antes presentado proponemos trabajar con tres categorías sustantivas que permitirán abordar la recepción de la teoría vigotskiana ${ }^{15}$ en el campo pedagógico, a saber:

a) Lecturas y usos de la categoría de Zona de Desarrollo Próximo (ZDP)

b) Lecturas y usos de la categoría de interiorización.

c) La vinculación de los usos de la teoría con el constructivismo como posición teórica.

${ }^{14}$ La reforma es tal en tanto constituyó una "alteración fundamental de la política educativas nacionales que pueden afectar al gobierno y administración del sistema educativo y escolar, y a su estructura y financiación, al currículum -contenidos, metodología y evaluación- al profesorado formación, selección o evaluación - y a la evaluación del sistema educativo" (Viñao; 2001, p. 26)

15 Cabe aclarar que no pretendemos agotar al análisis a través de las categorías propuestas. Volumen 9, Número Especial, Año 2009, ISSN 1409-4703 


\section{a) Lecturas y usos de la categoría de Zona de Desarrollo Próximo (ZDP)}

Se han identificado tres tipos de interpretaciones de la categoría de ZDP que permiten distintas lecturas acerca de las relaciones entre prácticas educativas y desarrollo:

$\checkmark$ La Zona de Desarrollo Próximo entendida como un "atributo subjetivo": a partir de esta interpretación se entiende a la Zona como una característica subjetiva, susceptible de ser evaluada o medida en el seno de interacciones específicas, como los psicodiagnósticos,

$\checkmark$ La Zona de Desarrollo Próximo entendida a partir del énfasis en las interacciones sociales. Esta segunda interpretación de uso de la ZDP guarda relación con la ponderación del efecto de las interacciones sociales sobre el desarrollo y el aprendizaje.

$\checkmark$ La Zona de Desarrollo Próximo y el giro contextualista: la tercer interpretación de la categoría de ZDP remite al cambio en la posibilidad de pensar y definir unidades de análisis del aprendizaje y el desarrollo no centradas en los individuos. En este punto, la tercer mirada sobre la Zona abre a lo que se denomina "giro contextualista". A partir del planteo vigotskiano busca el desplazamiento de la posición que implica sostener que la explicación última del desarrollo o del aprendizaje de una persona es atribuible con exclusividad a la persona misma. De este modo, el giro contextualista piensa a la persona en situación, esto es, como una posición mayor, que presupone la interacción con un mundo objetivo y social. La situación o contexto no constituye el exterior de los atributos de un individuo sino que tanto el desarrollo como el aprendizaje se dan en situación, y es la misma situación la que los explica (Baquero, 2001).

En esta última categoría se puede incluir la posición de Luis Moll (1993) en tanto sugiere pensar la Zona no como una característica exclusiva de la niñez o de la enseñanza, sino poner el foco en el sistema social que se crea activamente entre docentes y alumnos/as. Este sistema social implica una actividad colaborativa entre niños/as y adultos dentro de ámbitos culturales específicos. Recuérdese que cuando Vigotsky hacía referencia al "sistema social definido" estaba señalando hacia la organización social de la instrucción, y cómo esta organización ofrece una socialización especial al pensamiento de los niños/as (Daniels, 2001).

La concepción de la ZDP, como "sistema social", se define a partir de entender al sujeto comprometido en una actividad colaborativa. Moll sostiene, además, la necesidad de 
rechazar la definición de la Zona como enseñanza o evaluación de habilidades o subhabilidades separables. Para el autor

un problema en la aplicación del concepto de Zona al análisis de la instrucción en el aula es que el énfasis en la transferencia de conocimiento, y especialmente de habilidades, de aquellos que saben más a aquellos que saben menos, puede caracterizar cualquier práctica de enseñanza. (1990, p. 20)

Por lo tanto, es posible pensar que las prácticas escolares estándar no representan la idea original de Vigotsky, justamente debido a que suelen realizarse a través de tareas 0 ejercicios fragmentarios.

Resulta de utilidad, entonces, distinguir entre un uso descriptivo o explicativo de la categoría -vinculado a los procesos de desarrollo al interior de prácticas educativas, o en interacciones sociales diversas- de un uso prescriptivo, que podría adoptarse fundamentalmente en el campo pedagógico. En este sentido, se puede entender a la Zona como una herramienta para el análisis de sistemas de interacción definidos socialmente, ya que permite una aproximación a la naturaleza de ciertas transacciones sociales. Para Luis Moll, un análisis vigotskiano de la instrucción deberá poner el énfasis, centralmente, en la interdependencia entre personas adultas y los niños o niñas. La Zona puede ser pensada como una herramienta analítica necesaria para planificar la enseñanza y explicar sus resultados, ya que permite relacionar la perspectiva del desarrollo y la de la enseñanza. El supuesto implícito aquí es que tanto el desarrollo psicológico como la enseñanza se encuentran "socialmente implantados". Por lo tanto, y para entender ambos procesos, resulta necesario analizar la sociedad circundante y sus relaciones (Hedegaard, 1993).

\section{b) Lecturas y usos de la categoría de interiorización}

El giro contextualista en la interpretación de la ZDP genera, también, la posibilidad de diferenciarse de una lectura de la categoría de interiorización entendida como simple traspaso de habilidades. Esto supondría cierta escisión entre los planos social e individual y una reducción de los complejos procesos de desarrollo a la adquisición de habilidades o a la transferencia de paquetes de conocimiento de un exterior a un interior. 
Es fundamental para la práctica pedagógica detectar que la lectura de esta categoría puede llevar a tomar muy diferentes posiciones, entendiendo al alumnado como relativamente más o menos pasivo o activo en la situación de enseñanza y aprendizaje. Interesa pensar en el eje adquisición-transmisión: de manera sencilla y general se podría decir que las pedagogías basadas en la transmisión son aquellas que se apoyan en la idea de un sujeto ${ }^{16}$ más pasivo y las pedagogías basadas en la adquisición piensan en un sujeto más activo. James Wertsch (1995), sin embargo, complejiza esta mirada ya que postula dos tipos o modos de entender el proceso de interiorización en las que el sujeto es siempre activo, y el punto de interés pasa por el grado de identificación o resistencia con lo que se interioriza. En este sentido, diferencia la interiorización como "dominio", vinculada al "saber cómo" usar un modo de mediación con facilidad, de la interiorización como "apropiación", vinculada a una posición más activa del sujeto, que "hace propia" la herramienta.

Resulta fundamental cómo se conceptualizarán los recursos que pone a disposición la sociedad: si como una oferta fija o como un punto de inicio para la negociación (Daniels, 2001). Según Baquero (2002) las perspectivas denominadas contextualistas o situacionales pueden permitir abrir una agenda crítica de problemas sobre el desarrollo y las prácticas educativas. En este sentido, algunos autores como Rogoff (1993) prefieren hablar -en lugar de "interiorización"- de "apropiación participativa" de los sujetos en eventos o actividades culturales, un concepto que remite a la idea de sujeto activo constructor, donde sujeto y contexto están mutuamente vinculados. Esta categoría permite pensar en un proceso de transformación constructiva ${ }^{17}$. La postura de Rogoff busca de ese modo diferenciarse de una lectura de la interiorización como simple traspaso o internalización, donde lo social precede a lo individual, y buscar borrar la frontera entre lo externo y lo interno (Baquero, 2001). Similar posición sostiene Cazden (1991), quien también manifiesta la importancia de evitar una concepción mecánica de este concepto

16 Tomamos "sujeto" como una categoría clásica de la psicología, más allá del género.

17 "Con la guía de las personas que los rodean, los niños participan en actividades culturales que les permiten integrarse en su grupo social adoptando papeles que exigen destrezas específicas" (Rogoff, 1993, p.152). 


\section{c) La vinculación de los usos de la teoría con el constructivismo como posición teórica}

A partir de Hatano (1993), se pueden definir tres tipos de "extensiones" de esta teoría en el campo educativo a partir del análisis de las derivaciones pedagógicas de la perspectiva vigotskiana, y en relación con su carácter más o menos constructivista:

$\checkmark$ La extensión "estándar": vinculada con las primeras derivaciones pedagógicas de la teoría y privilegia las cuestiones relacionadas con la transmisión de contenidos o conocimientos, por sobre los aspectos constructivos de los procesos del desarrollo y el aprendizaje. Según Baquero (1998), esta extensión de la teoría parece proceder de una lectura unívoca de la categoría de ZDP, que la entendería como una suerte de andamiaje o práctica andamiada de la enseñanza, entendida de manera simplificada al modo de una mera "transmisión de habilidades". La aplicación estándar descuidaría los aspectos constructivos de los procesos de enseñanza y aprendizaje, poniendo el énfasis en formas de interacción asimétricas y verticales. Esta concepción de la adquisición del conocimiento basada en aspectos instruccionales, construida en base a la teoría de Vigotsky, se podría definir a partir de los siguientes aspectos:

- El conocimiento adquirido por el/la aprendiz es propiedad de quien enseña, y consiste en un set de habilidades o estrategias para la resolución de problemas. Quien enseña tiene como misión asignada por la sociedad esta transmisión de habilidades.

- El/la aprendiz resuelve junto al maestro/a algunos problemas específicos. Los maestros/as transmiten el conocimiento en una forma verbal codificada, y muestran cómo se resuelve el problema.

- A medida que el/la aprendiz se apropia del conocimiento, lo va resolviendo paso a paso, y el papel docente se vuelve cada vez menos importante

- Cuando el/la aprendiz puede resolver el problema por sus propios medios, se considera que el conocimiento ha sido transmitido exitosamente.

Según Hatano, esta concepción vigotskiana de la enseñanza y el aprendizaje es sólo una de las posibles, y está generalmente acompañada de algunos presupuestos empiristas: el/la aprendiz es básicamente un ser pasivo/a, no necesita comprender el significado de las 
herramientas que utiliza y aprende. Solo la interacción con el/la docente facilita la adquisición de conocimiento y aquel es siempre la única fuente de información y evaluación ${ }^{18}$.

$\checkmark$ La "extensión moderada": flexibiliza algunos aspectos y da lugar a interacciones múltiples, tanto como a posiciones más activas de los/las aprendices. Para el autor, si bien en estas "extensiones moderadas" de la teoría el trabajo cooperativo aumenta la capacidad de trabajo de aquellos, nunca la actividad va más allá, en definitiva, de la transmisión de habilidades de una persona adulta al aprendiz. La pregunta es entonces si esta adquisición de habilidades se debió a una genuina búsqueda de significados por parte de los alumnos, o si se debió al peso de la autoridad docente y la necesidad de aprenderlas a la hora de ser evaluados.

$\checkmark$ La "extensión radical": implica una genuina construcción de conocimiento por parte de las personas aprendices, en un marco de intercambio y restricciones socioculturales, que impulsa a quienes educan a buscar alternativas para la enseñanza. Esta concepción reemplazaría los presupuestos empiristas sobre la naturaleza del ser aprendiz, planteados más arriba, por los siguientes:

- Los aprendices son activos constructores de su conocimiento: toman la iniciativa en el aprendizaje e interactúan con personas de manera espontánea, y encuentran satisfacción en ambientes que favorezcan estos aspectos. Esta perspectiva se opone a la visión del aprendizaje como una transferencia o transmisión de modos o productos culturales del pensamiento.

- Quien está en lugar de aprendiz busca activamente comprender y habitualmente logra comprensiones. Esto quiere decir que además de activo es competente. La competencia está básicamente apoyada en su conocimiento previo, el cual le permite procesar la nueva información. Es incluso muy habitual que los/las aprendices puedan construir significados que vayan más allá de los otorgados por docentes.

- Esta construcción de conocimiento por parte de los aprendices se ve facilitada tanto por interacciones horizontales como verticales.

18 Según Ricardo Baquero (1998), si bien esta extensión estándar parece encontrarse principalmente en los primeros trabajos orientados por la teoría vigotskiana en el campo pedagógico, sería importante marcar que los trabajos sobre pedagogía de la escritura analizados por Cazden muestran que no se trató de un modo excluyente de utilizar la teoría. 
- El acceso a múltiples fuentes de información ayuda a la construcción del conocimiento, porque demasiada confianza en la respuesta autorizada del maestro/a suele reducir la motivación para construir conocimiento por propios medios.

- En la misma línea, se sostiene que el/la docente no debe ser la única fuente de validación de los saberes y conocimientos en el aula.

Según Hatano, entonces, estas características deberían ayudar a pensar y definir una perspectiva constructivista vigotskiana, que debería adoptar algunas ideas tales como:

- Los aprendizajes y enseñanzas en el contexto escolar están atravesados por aspectos normativos; por lo tanto, es función del docente establecer normas que impulsen el aprendizaje constructivo.

- Los aprendizajes y enseñanzas tienen limitaciones impuestas por las instituciones.

En este sentido, la creación de ZDP debe responder a la posibilidad de que adultos y aprendices puedan crear mutuamente formas de cooperación, a partir de las cuales los y las aprendices puedan crear significados que no podrían generar por sus propios medios. Esta búsqueda de sentido y significado por parte de los y las aprendices resulta crucial para pensar los procesos educativos.

\subsection{Recepción e idearios pedagógicos}

Ante lo expuesto nos preguntamos: ¿Cuál es el ideario pedagógico que, en las últimas dos décadas, se define en el medio local a partir de la teoría vigotskiana? Un relevamiento de diferentes publicaciones educativas de la Argentina durante el período 1990-2002 ${ }^{19}$ permite definir que este ideario está marcado, en primer término, por la mayor horizontalidad en las prácticas educativas frente al verticalismo que se impugna a las prácticas tradicionales con las que se discute.

19 El mismo, forma parte de la Tesis de Maestría de Andrés Gomel: "Una aproximación a las relaciones entre psicología y educación. Estudio sobre tres revistas educativas en la Argentina", llevada a cabo en la Universidad de San Andrés de la Argentina. Es fundamental indicar que en este relevamiento encontramos muchas menciones a categorías de la teoría vigotskiana, pero pocas menciones a obras específicas de Vigotsky. De este modo, reconstruimos algunos de los modos en los que se dio la recepción de esta teoría en el medio argentino, pero no la recepción de textos u obras específicos. 
Aparece, fundamentalmente, la importancia de la interacción social como elemento clave en los procesos de aprendizaje. $\mathrm{Y}$, en todos los casos, se enfatiza la centralidad del rol docente en los procesos de enseñanza y aprendizaje.

Vigotsky surge como autor pionero en cuanto a la importancia del trabajo en interacción con otras personas como base del desarrollo cognitivo. En diversos textos -como publicaciones científicas o de divulgación, o documentos oficiales- la sola mención de Vigotsky u otros autores de los ESC "autoriza" el trabajo con y la utilización de elementos tales como los aspectos referidos a "lo social" en los procesos de enseñanza y aprendizaje, o bien aquellos relacionados con el trabajo cooperativo, o entre pares, etc.

Otro elemento clave es el mayor énfasis que se observa referido a la centralidad del trabajo docente. Es posible pensar que esta centralidad del rol docente se enmarcaría, en cierta medida, en el constructivismo como marco amplio. Esto implicó una relectura que comienza a operarse sobre la lectura tradicional del rol, relectura que configura una revisión del mismo tal como había sido enmarcado según algunos seguidores de la teoría piagetiana.

La categoría de ZDP, por su parte, aparece como la sometida en mayor medida a deslizamientos de sentido, y ha sido en los últimos años objeto de múltiples interpretaciones y usos. Los primeros entre estos usos detectados en diferentes textos se pueden definir como más cercanos a fines diagnósticos. Estos usos -propios de fines de la década de 1980 y principios de la de 1990- evidencian también una concepción de la ZDP como un "espacio para el trabajo", definido de manera muy inespecífica, y como una categoría que permite poner de relieve la importancia de "lo social" en los procesos de aprendizaje.

Entendemos que las formas de plantear el trabajo en relación a la categoría de ZDP están en buena medida definidas por la influencia del Constructivismo como marco general de comprensión de los procesos de enseñanza y aprendizaje, proceso que como vimos se consolidaría a partir de la sanción de la Ley Federal de Educación y la implantación de la Reforma educativa. 
De este modo, se puede afirmar que la teoría sociohistórica en general, y esta categoría en particular, ingresaron en el medio local masivamente a partir de los textos propios de la Reforma y de revistas y libros (en muchos casos de autores españoles).

Es importante mencionar que en el período al que hacemos referencia, y aún en la actualidad, la categoría de ZDP ha funcionado como legitimadora de prácticas de aula de tipo horizontal. De este modo, la lectura de Vigotsky, y de esta categoría en particular, fundamentó la crítica a prácticas de tipo vertical, que quedaron asociadas a los fundamentos de la pedagogía más tradicional.

Otro elemento por considerar, vinculado con el anterior, es cómo la lectura de la ZDP llevó en muchos casos a igualar esta categoría, en el contexto argentino, con la de "andamiaje". Como es sabido, esta última categoría fue desarrollada por Bruner, Ross y Woods en 1976 tomando en consideración el planteo vigotskiano, pero sabemos que no se trata de categorías análogas punto a punto. Sin embargo, la lectura de publicaciones y documentos oficiales propios, fundamentalmente de la década de 1990, nos permite ver cómo en muchos casos ambas teorías parecen fundirse en una, perdiéndose en ese movimiento lo distintivo de cada una.

Liev Vigotsky entró a las instituciones educativas desde una lectura que transformó sus presupuestos teóricos en propuestas prescriptivas funcionales a la escuela. Interesa indicar que la indagación realizada por Marta Tenutto (2005) hace visible la inscripción que tiene Vigotsky en el discurso del docente.

\section{Conclusiones}

La teoría sociohistórica fue leída en muchos casos "en clave de reforma" y contribuyó a legitimar ciertas estrategias de trabajo áulico -como el trabajo colaborativo- y un nuevo lugar docente, de mayor importancia y centralidad. En este sentido, estuvo en línea con la "pedagogía centrada en el adulto" o "de sesgo adultocéntrico" (Baquero: 1998). De este modo una propuesta que podía ofrecer conceptos potentes, como los aportados por Vigotsky, fue en alguna medida capturada por las lógicas dicotómicas e instituidas del formato escolar, aquellas que habitualmente transforman "lo nuevo" en un polo de la tensión entre lo que "debe ser" y lo que no. En la actualidad, estos usos y lecturas de la teoría no Volumen 9, Número Especial, Año 2009, ISSN 1409-4703 
han perdido vigencia en las prácticas sino que, por el contrario, se han afirmado y hasta "naturalizado".

La teoría de la recepción resulta potente para analizar al autor en cuanto obra y desandar aquellas lecturas lineales, prescriptivas y carentes de texturas. Nos resulta indudable que estos usos vigotskianos, influenciados por la lectura realizada en clave constructivista, deberán ser revisados a partir de nuevas lecturas que permitan y autoricen novedosos y diversos modos de apropiación y utilización por parte de los actores del campo educativo.

\section{Agradecimientos}

Andrés Gomel y Marta Tenutto agradecen a la Universidad de San Andrés y la Fundación Lúminis la posibilidad que les han brindado al colaborar en su formación como becarios. Este hecho permitió la elaboración de las respectivas tesis y posibilitó la elaboración de este artículo.

\section{Referencias}

Baquero, Ricardo. (2001). La educabilidad bajo sospecha. Cuadernos de Pedagogía, IV (9), 71-85. Rosario: Editorial Homo Sapiens.

Baquero, Ricardo. (1998). Tensiones y paradojas en el uso de la Psicología Sociohistórica en educación. En Mario Carretero, José Antonio Castorina y Ricardo Baquero (comps.), Debates constructivistas. Buenos Aires: Aique.

Baquero, Ricardo y Margarita Limón. (2001). Introducción a la Psicología del aprendizaje. Bernal: Ediciones UNQ.

Caruso, Marcelo y Gabriela Fairstein. (1997). Las puertas del cielo. Hipótesis acerca de la recepción de la psicogénesis y el constructivismo de raíz piagetiana en el campo pedagógico argentino. En Puiggrós, A., Dictadura y utopías en la historia de la educación argentina (1955-1983). Buenos Aires: Galerna.

Castorina, José Antonio et al. (1996). Piaget- Vigotsky, contribuciones para replantear el debate. Buenos Aires: Paidós.

Cazden, Courtney. (1989). El discurso del aula. En M. Wittrock (Comp.) La investigación de la enseñanza. (pp. 627-709). Barcelona: Paidós: MEC. 
Cazden, Courtney. (1998). El discurso en el aula. El lenguaje de la enseñanza y del aprendizaje. Barcelona: Paidós, MEC.

Coll, César. (1991). Aprendizaje escolar y construcción del conocimiento. Barcelona: Paidós.

Daniels, Harry. (2001). Vigotsky y la pedagogía. Barcelona: Paidós.

Edwards, Derek y Neil Mercer. (1988): El desarrollo de la comprensión en el aula. Barcelona: Paidós.

Gomel, Andrés. (2008). Una aproximación a las relaciones entre psicología y educación. Estudio sobre tres revistas educativas en la Argentina. Tesis de Maestría. Universidad de San Andrés. Buenos Aires: Argentina.

Goodman, Yetta y Goodman, Ken. (1993). Vygotsky desde la perspectiva del lenguaje total. En Vygotsky y la educación. Connotaciones y aplicaciones de la Psicología Sociohistórica en la Educación. Buenos Aires: Aique.

Hatano, Giyoo. (1991). Time to Merge Vygotskian and Constructivist Conceptions of Knowledge Acquisition. En Forman, E., Minick, N y Addison Stone, C. (eds.) Contexts for Learning. Oxford: Oxford University Press.

Hedegaard, Marian. (1993). La Zona de Desarrollo Próximo como base para la enseñanza. En Vygotsky y la educación. Connotaciones y aplicaciones de la Psicología Sociohistórica en la Educación. Buenos Aires: Aique.

Moll, Luis. (1993): Vygotsky y la educación. Connotaciones y aplicaciones de a Psicología Sociohistórica en la Educación. Buenos Aires: Aique.

Newman, Denis, Griffin, Peg y Cole, Michael. (1991). La zona de construcción del conocimiento. Madrid: Morata.

Parrat-Dayan, Silvia. (1994). Piaget dans l'Ecole Libératrice: la dialectique de l'autre et du même. Archives de Psychologie, (62), 171-192. París

Parrat-Dayan, Silvia. (1998). Egocentrisme enfantin: concept structurel ou fonctionnel. Bulletin de Psychologie, tomo 51, 5, (437), 537-546. París: Sorbonne.

Parrat-Dayan, Silvia y Tryphon, Anastasia. (1998) Introduction. En Piaget, J. De la pèdagogie. Paris: O. Jacob.

Maldonado Mendoza, Fabio. (1994) Ejercicios de la epistemología. Madrid: Interciencia, 19 (2), 86-93. Disponible en http://www.interciencia.org/v19 02/ensayo.html.

Popper, Karl. (1985). Realismo y el objetivo de la ciencia: Post Scriptum a La lógica de la investigación científica (vl. 1, ed.preparada por W. W. Bartley III, Traducción de Marta Sansigre Vidal). Madrid: Editorial Tecnos.

Percia, Marcelo. (1994). Una subjetividad que se inventa. Buenos Aires, Lugar Editorial. 
Rogoff, Bárbara. (1993): Aprendices del pensamiento. El desarrollo cognitivo en el contexto social. Barcelona: Paidós.

Tenutto, Marta. (2005). Los significados atribuidos por los profesores de profesorado al Constructivismo. Un estudio de caso. Tesis para optar por el grado de Maestría en Educación con Especialización en Gestión, Universidad de San Andrés, Buenos Aires, Argentina.

Tudge, Jonathan. (1993). Vigotsky, la zona de desarrollo próximo y la colaboración entre pares: connotaciones para la práctica en el aula. En Vygotsky y la educación. Connotaciones y aplicaciones de la Psicología Sociohistórica en la Educación. Buenos Aires: Aique.

Vigotsky, Lev. (1979). El desarrollo de procesos psicológicos superiores. Barcelona: Grijalbo.

Vigotsky, Lev. (1979). Pensamiento y lenguaje. Buenos Aires: La Pléyade.

Vigotsky, Lev. (2001). Psicología Pedagógica (1a ed. 1926). Buenos Aires: Aique.

Vigotsky, Lev. (1991). El método instrumental en psicología. En Obras Escogidas (Tomo 1, pp. 65-70). Barcelona: Visor.

Vigotsky, Lev. (1991). El problema del desarrollo de las funciones psíquicas superiores. En Obras escogidas (Tomo 3, pp. 11-46). Barcelona: Visor.

Vigotsky, Lev. (1991). Génesis de las funciones psíquicas superiores. En Obras escogidas (Tomo 3, pp. 139-168). Barcelona: Visor.

Vigotsky, Lev. (1991). La conciencia como problema de la psicología del comportamiento. Obras Escogidas (Tomo 1, pp. 39-60). Barcelona: Visor.

Vigotsky, Lev. (1991). La psicología y la teoría de la localización de las funciones psíquicas. En Obras escogidas (Tomo 3). Barcelona: Visor.

Vigotsky, Lev. (1991). La psique, la conciencia y el inconsciente En Obras Escogidas (Tomo 1, pp. 95-110). Barcelona: Visor.

Vigotsky, Lev. (1991). Los métodos de investigación reflexológicos y Psicológicos. En Obras Escogidas (Tomo 1). Barcelona: Visor.

Vigotsky, Lev. (1991). Prefacio al libro de A.N. Leóntiev: Desarrollo de la memoria. En Obras escogidas: problemas teóricos y metodológicos de la psicología. Barcelona: Visor.

Vigotsky, Lev. (1991). Prólogo al libro de A.F. Lazurski: Psicología general y experimental. En Obras Escogidas (Tomo 1, pp. 23-38). Barcelona: Visor.

Vigotsky, Lev. (1991) Sobre el artículo de K. Koffka: La introspección y el método de la psicología. Obras Escogidas (Tomo 1, pp. 61-64).). Barcelona: Visor. 
Vigotsky, Lev. (1991). Estructura de las funciones psíquicas superiores. En Obras escogidas (Tomo 3, pp. 121-138). Barcelona: Visor.

Wertsch, James. (1991). Voices of the mind. Cambridge: Harvard University Press.

Wertsch, James. (1995). Vigotsky y la formación social de la mente. Barcelona: Paidós. 